\title{
Esquemas cognitivos disfuncionales y dependencia emocional en mujeres con y sin violencia en la relación de pareja de la ciudad de Lima
}

Early maladaptive schemas and emotional dependence in battered women and no battered women in the city of Lima

\author{
Rosa Huerta ${ }^{1}$, Natalia Ramírez, Jessica Ramos, Lourdes Murillo, César Falcón, \\ Miguel Misare, José Sánchez \\ Facultad de Psicología, UNMSM, Lima - Perú
}

RECIBIDO 30/06/2016, AcEPTADO 26/08/2016

\begin{abstract}
RESUMEN
En el estudio se sometió al análisis las variables esquemas cognitivos disfuncionales tempranos y dependencia emocional en función a la presencia o no de violencia en una muestra de 385 mujeres con y sin violencia en la relación de pareja, a quienes se les administró una ficha de datos sociodemográficos, el Cuestionario de Esquemas de Young integrado por 18 esquemas y el Inventario de Dependencia Emocional. Se encontró que existe una correlación muy significativa y positiva entre las dimensiones y los esquemas disfuncionales tempranos con los factores de la dependencia emocional, agravada por la presencia de violencia en la relación de pareja (sig. $=0.01$ ), lo cual es un indicador de riesgo latente en la muestra examinada porque facilita la permanencia en una relación de pareja disfuncional y violenta así como alto riego en su salud mental. Se encontró además mayor relación entre la dependencia emocional con los esquemas; cuando hay violencia las dimensiones están más afectadas. Asimismo, se hallaron diferencias significativas entre ambos grupos. Es la presencia de violencia en la relación de pareja la que activa los esquemas y produce síndromes y trastornos en la salud mental de las mujeres.
\end{abstract}

Palabras clave: Esquemas cognitivos, dependencia emocional, violencia en la relación de pareja y mujeres víctimas de maltrato.

\begin{abstract}
The study submitted to the analysis of the following variables: early maladaptive schemas and emotional dependence in function to the presence or not of violence in a sample of 385 women battered women and no battered, who were given a sociodemographic survey, the Young Schema Questionnaire (YSQ-S3) composed of 18 schemes and the Emotional Dependence Inventory. It was found that there is a significant and positive correlation between dimensions and early maladaptive schemes with factors of emotional dependency, compounded by the
\end{abstract}

1 Docente Facultad de Psicología de la UNMSM. E-mail: huertarosel@hotmail.com 
presence of violence in the relationship (sig. $=0.01$ ) which is an indicator of latent risk in the sample examined because it facilitates the permanence in a dysfunctional and violent relationship with a partner as well as a high risk of their mental health. In addition, mayor relationship between emotional dependence and early maladaptive schemes, when there is violence Dimensions of schemes are more affected. It was also found significant differences between the two groups. The presence of violence in the couple relationship activates the early maladaptive schemes and produce syndromes and mental disorders in the women.

Keywords: Early maladaptive schemas, emotional dependence, violence in couple relationship and battered women.

\section{INTRODUCCIÓN}

Actualmente, muchos estudios muestran la importancia de las cogniciones de las mujeres maltratadas, pero no existen aún estudios que directamente examinen si los esquemas cognitivos median o moderan la influencia del maltrato de la pareja en los síntomas depresivos. Lerner y Kennedy (2000), citado en Calvete, E., Corral, S. y Estévez, A. (2007), encontraron que la experiencia de maltrato durante la infancia está unida a las distorsiones en los esquemas cognitivos adultos y esos esquemas cognitivos, en cambio, estaban asociados con la aceptación de la violencia en las relaciones de violencia en pareja. McGinn y Young (1996) describieron que los esquemas cognitivos se activan por los ambientes estresantes que son relevantes a cada esquema particular. Cuando se activan, los esquemas generan emociones muy intensas en la persona y conducen directa o indirectamente a problemas psicológicos tales como depresión, sentimientos de soledad, relaciones destructivas, adicciones al alcohol y drogas, entre otros.

Las situaciones traumáticas, como ser víctima de maltrato por parte de la pareja, podrían alterar los esquemas cognitivos sobre sí mismas y el mundo previos a la violencia y cambiarlos por cogniciones negativas. Es más, una variedad de estudios ha encontrado cambios profundos en las creencias y esquemas de mujeres maltratadas (Arias y Pape, 1999; Dutton, Burghart, Perrin, Chrestman, y Halle, 1994). Tanto la violencia física, psicológica y sexual se asociaron positivamente a los esquemas cognitivos y estos se asociaron positivamente a los síntomas depresivos (Roman, I.; 2011).

Los esquemas referidos a Desconexión y Rechazo y el referido a temas de abandono, abuso e imperfección, pueden ser activados cuando las mujeres son víctimas de violencia extrema, tal como se ha comprobado en niños/as que han sufrido abuso (Berstein, 2002). El esquema de abuso implica la creencia de que las personas queridas abusarán y harán daño, y el esquema de imperfección se refiere a creencias de fallo, inadecuación personal y culpabilidad, tan frecuentemente encontradas en víctimas de maltrato (Dutton, 1992).

Por otro lado, el ser testigos presenciales de violencia en el hogar favorece, desde tempranas edades, la instauración de creencias y esquemas mentales disfuncionales en las víctimas de esta, afectando su salud mental y las relaciones interpersonales 
de todos los miembros de la familia. Igualmente, el hecho de estar involucrado en la situación de violencia intrafamiliar en la edad adulta favorece la activación de dichos esquemas y su respectivo mantenimiento.

La dependencia emocional está asociada con episodios de depresión ante las pérdidas (muerte de cónyuge o familiar, separación o divorcio, enfermedad o abandono del cónyuge) y con maltrato en la relación de pareja. Los dependientes emocionales sufren sintomatología ansiosa y depresión leve durante gran parte de sus vidas, basada en su falta de autoestima, en sus problemas de pareja y en su continuo miedo al abandono por parte de la otra persona.

En relación con la dependencia afectiva, Young (1999) plantea que quienes tienen el esquema de "desconfianza/abuso" presentan la sensación de que sus necesidades afectivas no serán satisfechas de modo predecible, perciben su entorno como lastimador, y consideran que el daño sufrido es intencional por parte de los demás o es consecuencia de una injustificada y extrema negligencia.

Violencia en la relación de pareja, definida también como violencia doméstica, es conceptualizada como "un patrón de conductas abusivas, que incluye un amplio rango de maltrato físico, sexual y/o psicológico y que es ejercido por una persona en contra de otra, en el contexto de una relación íntima, con el objeto de ganar o mantener el abuso de poder, control y autoridad sobre ella" (Walker, 1999, p.23).

Los esquemas cognitivos disfuncionales son definidos como procesadores de información, estructuras estables y duraderas resistentes al cambio que se inician en la infancia y se desarrollan durante la vida del individuo. Asimismo representan las conductas y pensamientos disfuncionales y experiencias adquiridas en el entorno (Young, 1999). Según el modelo teórico de Young (1999) el origen de los esquemas cognitivos está en la infancia, siendo clave las experiencias tempranas con los padres y madres. Young (1999) plantea 18 esquemas cognitivos organizados en cinco grandes dominios o categorías. De hecho, cuando la víctima sufre una experiencia prolongada de maltrato por parte de su pareja, sus pensamientos sobre sí misma y su autoestima pueden llegar a ser enormemente negativos, aumentando el riesgo de aparición de depresión. Calvete, Estévez y Corral (2007), en un estudio reciente, demostraron que varios esquemas disfuncionales actuaban como mediadores entre la violencia por parte de la pareja y la depresión.

La dependencia emocional es definida como la dimensión disfuncional de un rasgo de personalidad, que consiste en la necesidad extrema de orden afectiva que una persona siente hacia otra, su pareja, a lo largo de sus diferentes relaciones de pareja (Castello, 2000, 2005).

Los dependientes emocionales presentan apego ansioso. Establecen relaciones de sumisión, anhelo por preservarlas a cualquier coste, o el emparejamiento con personas narcisistas y explotadoras, es decir, relaciones autodestructivas. Así mismo presentan pobre autoestima y un autoconcepto negativo no ajustado a la realidad, problemas de ansiedad y/o depresión y un cierto desprecio por sí mismo al ser consciente de que se está arrastrando ante alguien que no solo no lo ama sino que incluso lo maltrata, (García, 2014). 
Para el presente estudio se planteó como objetivos, analizar la relación entre las dimensiones de los esquemas cognitivos disfuncionales y la dependencia emocional en mujeres víctimas y no víctimas de violencia en la relación de pareja de la ciudad de Lima; asimismo, comparar las diferencias en las dimensiones de los esquemas cognitivos disfuncionales y la dependencia emocional entre las mujeres con y sin violencia en la relación de pareja de la ciudad de Lima. Otro objetivo ha sido adaptar el Cuestionario de Esquemas Cognitivos de Young, (YSQ-S3).

\section{MÉTODO}

El presente estudio es de tipo sustantivo, sistematiza procedimientos dentro del método correlacional. Adicionalmente, la contrastación nos permitió conocer la intensidad y dirección de la relación entre los esquemas cognitivos disfuncionales y la dependencia emocional en mujeres con y sin violencia en la relación de pareja. El diseño fue descriptivo correlacional, transversal, los datos fueron recogidos en un solo momento (Hernández, Fernandez y Baptista, 2010).

\section{MUESTRA}

La muestra quedó constituida por 385 mujeres, que se fueron clasificando por edad, luego según la entrevista por la presencia o no de violencia, por el tipo de violencia. Las edades fueron entre 14 y 60 años, predominando la edad de 21 a 35 años (46.5\%) tanto en el grupo con violencia (204), como el de sin violencia (181).

Al revisar el tipo de violencia, según la declaración de las mujeres víctimas, se aprecia un predominio de violencia física en todos los grupos de edad con una mayor frecuencia en el grupo de 21 a 35 seguido del 36 a 45 años.

\section{TÉCNICAS E INSTRUMENTOS DE RECOLECCIÓN DE DATOS}

Para la recolección de datos se determinaron los establecimientos de salud y las instituciones de la ciudad de Lima que atienden esta problemática. La aplicación de los instrumentos se realizó de manera individual. Las escalas que, a continuación, se describen (previamente, se revisó la traducción en el caso del Cuestionario YSQ-S3) fueron adaptadas lingüísticamente.

\section{INSTRUMENTOS}

Ficha de datos sociodemográficos: Elaborada por el investigador. Aplicada de manera individual. Permite recoger datos como lugar de procedencia, edad, grado de instrucción, existencia o no de violencia, así como los tipos de violencia en los que se encuentran involucrados. 
Cuestionario de Esquemas de Young (YSQ-S3) de Jeffrey Young. Es un instrumento de indagación aplicable a sujetos a partir de los 16 años. Consta de 90 reactivos, cada uno de ellos está estructurado en una escala tipo Likert por seis enunciados, de los cuales el sujeto tiene que seleccionar el que mejor describa su forma de evaluar una situación. Evalúa los esquemas cognitivos disfuncionales tempranos o denominado también esquemas maladaptativos tempranos. Mide 18 factores agrupados en cuatro dimensiones.

Se ha revisado la traducción y se ha realizado la adaptación lingüística, procediéndose a obtener la validez de constructo y la confiabilidad llegando según el alfa de Cronbach a $\mathrm{r}=0.97$. Asimismo, Castrillon et al. (2005) elaboran una versión de este cuestionario y obtienen un alfa de Cronbach de 0.95. A continuación, se detallan las 5 dimensiones y los 18 esquemas:

Tabla 1. Dimensiones y Esquemas cognitivos disfuncionales tempranos

\begin{tabular}{|c|c|c|}
\hline Dimensión I: Desconexión y Rechazo & Dimensión IV: & $\begin{array}{c}\text { Direccionalidad hacia los } \\
\text { demás }\end{array}$ \\
\hline Esquema 1: Abandono/inestabilidad & & Esquema 12: Subyugación \\
\hline Esquema 2: Desconfianza/abuso & & Esquema 13: Autosacrificio \\
\hline Esquema3: Privación emocional & & $\begin{array}{l}\text { Esquema 14: Búsqueda de } \\
\text { aprobación }\end{array}$ \\
\hline \multicolumn{3}{|l|}{ Esquema 4: Imperfección/vergüenza } \\
\hline \multicolumn{3}{|l|}{ Esquema 5: Aislamiento social/alienación } \\
\hline Dimensión II: Deterioro en autonomía y ejecución: & Dimensión V: & Sobrevigilancia e inhibición \\
\hline Esquema 6:Dependencia/incompetencia & & $\begin{array}{l}\text { Esquema 15: Negatividad/ } \\
\text { Pesimismo }\end{array}$ \\
\hline $\begin{array}{l}\text { Esquema } 7 \text { : Vulnerabilidad al peligro } \\
\text { o a la enfermedad }\end{array}$ & & $\begin{array}{l}\text { Esquema 16: Inhibición } \\
\text { emocional: }\end{array}$ \\
\hline Esquema 8: Apego confuso y/o inmaduro & & $\begin{array}{l}\text { Esquema 17: Metas } \\
\text { inalcanzables/ hipercrítica }\end{array}$ \\
\hline Esquema 9: Fracaso & & Esquema 18: Castigo \\
\hline \multicolumn{3}{|l|}{ Dimensión III: Límites alterados } \\
\hline \multicolumn{3}{|l|}{ Esquema 10: Grandiosidad/Autorización } \\
\hline $\begin{array}{l}\text { Esquema 11: Insuficiente auto-control/ } \\
\text { autodisciplina }\end{array}$ & & \\
\hline
\end{tabular}

Inventario de Dependencia Emocional de Aiquipa, J. (IDE). Es un instrumento aplicable a partir de los 16 años. Consta de 49 reactivos, cada ítem valorado en una escala Likert de seis puntos que va desde uno hasta seis. En nuestro medio fue construida por Aiquipa, J. (2012). El IDE permite observar la dependencia emocional; obteniendo un coeficiente de confiabilidad de 0.91 . 


\section{RESULTADOS.}

1. Relación entre las dimensiones de los esquemas cognitivos disfuncionales y la dependencia emocional en mujeres víctimas y no víctimas de violencia en la relación de pareja de la ciudad de Lima en la muestra general. Los datos obtenidos de ambas variables se correlacionaron con el coeficiente Rho de Spearman encontrando relaciones significativas y positivas entre las dimensiones de los esquemas cognitivos disfuncionales con las dimensiones de la dependencia emocional.

Existe una relación positiva y significativa entre las 5 dimensiones de los esquemas cognitivos disfuncionales con la dependencia emocional total y con todos sus factores en la muestra total, predominando la dimensión 1, desconexión y rechazo; la dimensión 2, deterioro en autonomía y ejecución y la dimensión 5 sobrevigilancia e inhibición $(\mathrm{r}=0.678, \mathrm{r}=0.679$ y r $=0.660$ respectivamente $)$. Asimismo se aprecia correlación con las dimensiones 3 , límites alterados y la 4, direccionalidad a los demás, lo cual nos permite señalar que la presencia de esquemas tempranos disfuncionales incrementan la dependencia emocional. Ello suele estar determinado por necesidades emocionales nucleares insatisfechas durante la infancia, esquemas que van a influir en el procesamiento de sus experiencias posteriores, poniendo en riesgo la salud mental de la mujer pudiendo presentar ansiedad, depresión, trastornos psicosomáticos entre otros. Tal como se observa en la Tabla 2.

Tabla 2. De Coeficientes de correlación de las dimensiones de la Esquemas cognitivos con la Dependencia Emocional. Coeficiente de correlación - Rho de Spearman: Muestra Total.

\begin{tabular}{|c|c|c|c|c|c|c|c|c|c|}
\hline \multicolumn{10}{|c|}{ Dependencia emocional } \\
\hline $\begin{array}{l}\text { Dimensiones } \\
\text { Esquemas } \\
\text { disfuncionales }\end{array}$ & & $\begin{array}{c}\text { Dependencia } \\
\text { Total }\end{array}$ & $\begin{array}{l}\text { Miedo a } \\
\text { la ruptura }\end{array}$ & $\begin{array}{c}\text { Miedo e } \\
\text { intolerancia } \\
\text { a la soledad }\end{array}$ & $\begin{array}{l}\text { Prioridad } \\
\text { de la pareja }\end{array}$ & $\begin{array}{c}\text { Necesidad } \\
\text { de acceso a } \\
\text { la pareja }\end{array}$ & $\begin{array}{c}\text { Deseos de } \\
\text { exclusividad }\end{array}$ & $\begin{array}{l}\text { Subordinación } \\
\text { y sumisión }\end{array}$ & $\begin{array}{c}\text { Deseos de } \\
\text { control y } \\
\text { dominio }\end{array}$ \\
\hline $\begin{array}{l}\text { Dimensión } 1 \\
\text { Desconexión y } \\
\text { rechazo }\end{array}$ & Total &, $678^{* *}$ &, $633^{* *}$ &, $665^{* *}$ &, $646^{* *}$ &, $483^{* *}$ &, $575^{* *}$ &, $550^{* *}$ &, $631^{* *}$ \\
\hline $\begin{array}{l}\text { Dimensión } 2 \\
\text { Deterioro en } \\
\text { autonomía y } \\
\text { ejecución }\end{array}$ & Total &, $679^{* *}$ &, $646^{* *}$ &, $662^{* *}$ &, $651^{* *}$ &, $462^{* *}$ &, $591^{* *}$ &, $508^{* *}$ &, $635^{* *}$ \\
\hline $\begin{array}{l}\text { Dimensión } 3 \\
\text { Límites } \\
\text { alterados }\end{array}$ & Total &, $616^{* *}$ &, $562^{* *}$ &, $559^{* *}$ &, $591^{* *}$ &, $500^{* *}$ &, $509^{* *}$ &, $534^{* *}$ &, $568^{* *}$ \\
\hline $\begin{array}{l}\text { Dimensión } 4 \\
\text { Direccionalidad } \\
\text { hacia los demás }\end{array}$ & Total &, $562^{* *}$ &, $409^{* *}$ &, $503^{* *}$ &, $507^{* *}$ &, $525^{* *}$ &, $484^{* *}$ &, $499^{* *}$ &, $566^{* *}$ \\
\hline $\begin{array}{l}\text { Dimensión } 5 \\
\text { Sobrevigilancia } \\
\text { e inhibición }\end{array}$ & Total &, $660^{* *}$ &, $553^{* *}$ &, $632^{* *}$ &, $597^{* *}$ &, $529^{* *}$ &, $558^{* *}$ &, $553^{* *}$ &, $633^{* *}$ \\
\hline
\end{tabular}


$\mathrm{Al}$ revisar la relación entre los 18 esquemas con los 7 factores de la dependencia emocional, encontramos correlación positiva y significativa entre todos ellos, existiendo mayor correlación con los esquemas de desconfianza y abuso $(\mathrm{r}=0.672)$, privación emocional $(\mathrm{r}=0.632)$, fracaso $(\mathrm{r}=0.630)$, negatividad/ pesimismo $(\mathrm{r}=0.621)$ y apego confuso $\mathrm{y} / \mathrm{o}$ inmaduro $(\mathrm{r}=0.620)$. De la misma forma, los factores de dependencia emocional asociados son miedo e intolerancia a la soledad, miedo a la ruptura, prioridad de la pareja y deseos de control de la pareja; los cuales ponen de manifiesto que la mujer considera, en la muestra total, que sus necesidades de seguridad, aceptación y respeto no serán satisfechas, así como temor a la separación, quedarse con la pareja a pesar de sentirse insegura otorgándole prioridad sobre las otras personas a fin de asegurar su permanencia, tal como se observa en la Tabla 3.

Tabla 3. De Coeficientes de correlación de las dimensiones de la Esquemas cognitivos con la Dependencia Emocional. Coeficiente de correlación - Rho de Spearman. MuestraTotal.

\begin{tabular}{|c|c|c|c|c|c|c|c|c|}
\hline $\begin{array}{l}\text { Esquemas } \\
\text { cognitivos disfuncionales }\end{array}$ & $\begin{array}{l}\text { Dependencia } \\
\text { Total }\end{array}$ & $\begin{array}{l}\text { Miedo } \\
\text { a la } \\
\text { ruptura }\end{array}$ & $\begin{array}{c}\text { Miedo e } \\
\text { intolerancia } \\
\text { a la soledad }\end{array}$ & $\begin{array}{c}\text { Prioridad } \\
\text { de la } \\
\text { pareja }\end{array}$ & $\begin{array}{c}\text { Necesidad de } \\
\text { acceso a la } \\
\text { pareja }\end{array}$ & $\begin{array}{l}\text { Deseos de } \\
\text { exclusividad }\end{array}$ & $\begin{array}{l}\text { Subordinación } \\
\text { y sumision }\end{array}$ & $\begin{array}{c}\text { Deseos de } \\
\text { control y } \\
\text { dominio }\end{array}$ \\
\hline Total &, $694^{* *}$ &, $610^{* *}$ &, $662^{* *}$ &, $650^{* *}$ &, $534^{* *}$ &, $590^{* *}$ &, $570^{* *}$ &, $660^{* *}$ \\
\hline Abandono / Inestabilidad &, $552^{* *}$ &, $564^{* *}$ &, $562^{* *}$ &, $538^{* *}$ &, $343^{* *}$ &, $415^{* *}$ &, $460^{* *}$ &, $520^{* *}$ \\
\hline Desconfianza / Abuso &, $672^{* *}$ &, $566^{* *}$ &, $655^{* *}$ &, $612^{* *}$ &, $525^{* *}$ &, $573^{* *}$ &, $593^{* *}$ &, $608^{* *}$ \\
\hline Privación emocional &, $632^{* *}$ &, $518^{* *}$ &, $591^{* *}$ &, $584^{* *}$ &, $535^{* *}$ &, $546^{* *}$ &, $516^{* *}$ &, $608^{* *}$ \\
\hline Imperfección / vergüenza &, $547^{* *}$ &, $539^{* *}$ &, $534^{* *}$ &, $525^{* *}$ &, $359^{* *}$ &, $486^{* *}$ &, $434^{* *}$ &, $508^{* *}$ \\
\hline $\begin{array}{l}\text { Aislamiento social / } \\
\text { Alienación. }\end{array}$ &, $577^{* *}$ &, $630^{* *}$ &, $585^{* *}$ &, $569^{* *}$ &, $339^{* *}$ &, $518^{* *}$ &, $421^{* *}$ &, $525^{* *}$ \\
\hline $\begin{array}{l}\text { Dependencia / } \\
\text { Incompetencia }\end{array}$ &, $597^{* *}$ &, $647^{* *}$ &, $630^{* *}$ &, $592^{* *}$ &, $329^{* *}$ &, $524^{* *}$ &, $394^{* *}$ &, $536^{* *}$ \\
\hline $\begin{array}{l}\text { Vulnerabilidad al peligro o } \\
\text { a la enfermedad }\end{array}$ &, $571^{* *}$ &, $576^{* *}$ &, $571^{* *}$ &, $587^{* *}$ &, $331^{* *}$ &, $488^{* *}$ &, $419^{* *}$ &, $534^{* *}$ \\
\hline $\begin{array}{l}\text { Apego confuso y/o } \\
\text { inmaduro }\end{array}$ &, $620^{* *}$ &, $535^{* *}$ &, $578^{* *}$ &, $584^{* *}$ &, $472^{* *}$ &, $561^{* *}$ &, $481^{* *}$ &, $596^{* *}$ \\
\hline Fracaso &, $630^{* *}$ &, $575^{* *}$ &, $591^{* *}$ &, $566^{* *}$ &, $497^{* *}$ &, $537^{* *}$ &, $520^{* *}$ &, $597^{* *}$ \\
\hline $\begin{array}{l}\text { Grandiosidad / } \\
\text { Autorización }\end{array}$ &, $610^{* *}$ &, $621^{* *}$ &, $594^{* *}$ &, $596^{* *}$ &, $414^{* *}$ &, $508^{* *}$ &, $479^{* *}$ &, $553^{* *}$ \\
\hline $\begin{array}{l}\text { Insuficiente autocontrol/ } \\
\text { auto disciplina }\end{array}$ &, $516^{* *}$ &, $418^{* *}$ &, $437^{* *}$ &, $484^{* *}$ &, $490^{* *}$ &, $429^{* *}$ &, $489^{* *}$ &, $479^{* *}$ \\
\hline Subyugación &, $540^{* *}$ &, $451^{* *}$ &, $508^{* *}$ &, $505^{* *}$ &, $444^{* *}$ &, $477^{* *}$ &, $428^{* *}$ &, $541^{* *}$ \\
\hline Auto sacrificio &, $454^{* *}$ &, $283^{* *}$ &, $394^{* *}$ &, $385^{* *}$ &, $494^{* *}$ &, $381^{* *}$ &, $423^{* *}$ &, $458^{* *}$ \\
\hline Búsqueda de aprobación &, $529^{* *}$ &, $395^{* *}$ &, $470^{* *}$ &, $483^{* *}$ &, $470^{* *}$ &, $457^{* *}$ &, $493^{* *}$ &, $529^{* *}$ \\
\hline Negatividad / Pesimismo &, $621^{* *}$ &, $520^{* *}$ &, $589^{* *}$ &, $557^{* *}$ &, $494^{* *}$ &, $531^{* *}$ &, $528^{* *}$ &, $599^{* *}$ \\
\hline Inhibición emocional &, $567^{* *}$ &, $443^{* *}$ &, $529^{* *}$ &, $523^{* *}$ &, $455^{* *}$ &, $489^{* *}$ &, $467^{* *}$ &, $545^{* *}$ \\
\hline $\begin{array}{l}\text { Normas inalcanzables / } \\
\text { hipercrítica }\end{array}$ &, $590^{* *}$ &, $510^{* *}$ &, $577^{* *}$ &, $521^{* *}$ &, $470^{* *}$ &, $488^{* *}$ &, $501^{* *}$ &, $566^{* *}$ \\
\hline Castigo &, $571^{* *}$ &, $514^{* *}$ &, $559^{* *}$ &, $513^{* *}$ &, $475^{* *}$ &, $486^{* *}$ &, $474^{* *}$ & $526^{* *}$ \\
\hline
\end{tabular}

**. La correlación es significativa al nivel 0,01 (bilateral).

* La correlación es significativa al nivel 0,05 (bilateral). 
2. Relación entre las dimensiones de los esquemas cognitivos disfuncionales y la dependencia emocional en mujeres víctimas y no víctimas de violencia en la relación de pareja de la ciudad de Lima. Los datos obtenidos se correlacionaron con el coeficiente Rho de Spearman, encontrando relaciones positivas y significativas entre las dimensiones de los esquemas cognitivos disfuncionales y los factores de la dependencia emocional.

Al analizar los datos en el grupo de mujeres con violencia se encuentra una relación muy significativa (0.01) entre las dimensiones de los esquemas cognitivos disfuncionales tempranos y la dependencia emocional total como se puede observar en la Tabla 4. Al analizar cada esquema con cada factor de la dependencia emocional destacan las relaciones entre los 7 factores de la dependencia emocional con la dimensión 2 , deterioro de la autonomía y ejecución $(\mathrm{r}=0.733)$; con la dimensión 1 desconexión y rechazo $(\mathrm{r}=0.715)$; con la dimensión 5 , sobrevigilancia e inhibición ( $\mathrm{r}=0.700)$; seguidas de la relación con la dimensión 3, límites alterados $(\mathrm{r}=0.674)$ y la dimensión 4 , direccionalidad ante los demás $(\mathrm{r}=0.632)$. En el grupo sin violencia también encontramos una relación que va entre 0.471 y 0.584 , la cual es menor y significativa al 0.01 . Tales resultados nos permiten reforzar lo hallado en la muestra total de una relación positiva entre ambas variables, agravada por la presencia de violencia en la relación de pareja. Así, el estar inserto en una relación de violencia es un factor de riesgo para su salud mental y su salud en general que favorece la activación de los esquemas, como se observa en la Tabla 4.

Tabla 4. De Coeficientes de correlación de las dimensiones de la Esquemas cognitivos con la Dependencia Emocional. Coeficiente de correlación - Rho de Spearman

$($ Total $=385$, Violencia $=204$ y No violencia $=181)$

\begin{tabular}{|c|c|c|c|c|c|c|c|c|c|}
\hline \multicolumn{10}{|c|}{ Dependencia emocional } \\
\hline \multicolumn{2}{|l|}{$\begin{array}{l}\text { Esquemas } \\
\text { cognitivos } \\
\text { disfuncionales }\end{array}$} & \multirow{2}{*}{$\begin{array}{c}\begin{array}{c}\text { Dependencia } \\
\text { Total }\end{array} \\
, 678^{* *}\end{array}$} & \multirow{2}{*}{$\begin{array}{c}\begin{array}{c}\text { Miedo } \\
\text { a la } \\
\text { ruptura }\end{array} \\
, 633^{* *}\end{array}$} & \multirow{2}{*}{$\begin{array}{c}\begin{array}{c}\text { Miedo e } \\
\text { intolerancia } \\
\text { a la soledad }\end{array} \\
, 665^{* *}\end{array}$} & \multirow{2}{*}{$\begin{array}{c}\begin{array}{c}\text { Prioridad } \\
\text { de la } \\
\text { pareja }\end{array} \\
, 646^{* *}\end{array}$} & \multirow{2}{*}{$\begin{array}{c}\begin{array}{c}\text { Necesidad } \\
\text { de acceso a } \\
\text { la pareja }\end{array} \\
, 483^{* *}\end{array}$} & \multirow{2}{*}{$\begin{array}{c}\begin{array}{c}\text { Deseos de } \\
\text { exclusividad }\end{array} \\
, 575^{* *}\end{array}$} & \multirow{2}{*}{$\begin{array}{l}\begin{array}{l}\text { Subordinación } \\
\text { y sumisión }\end{array} \\
, 550^{* *}\end{array}$} & \multirow{2}{*}{$\begin{array}{c}\begin{array}{c}\text { Deseos de } \\
\text { control y } \\
\text { dominio }\end{array} \\
, 631^{* *}\end{array}$} \\
\hline Dimensión 1 & Total & & & & & & & & \\
\hline \multirow[t]{2}{*}{$\begin{array}{l}\text { Desconexión y } \\
\text { rechazo }\end{array}$} & Violencia &, $715^{* *}$ &, $661^{* *}$ &, $701^{* *}$ &, $693^{* *}$ &, $547^{* *}$ &, $646^{* *}$ &, $611^{* *}$ &, $675^{* *}$ \\
\hline & No violencia &, $584^{* *}$ &, $492^{* *}$ &, $566^{* *}$ &, $492^{* *}$ &, $441^{* *}$ &, $441^{* *}$ &, $432^{* *}$ &, $522^{* *}$ \\
\hline Dimensión 2 & Total &, $679^{* *}$ &, $646^{* *}$ &, $662^{* *}$ &, $651^{* *}$ &, $462^{* *}$ &, $591^{* *}$ &, $508^{* *}$ &, $635^{* *}$ \\
\hline \multirow{2}{*}{$\begin{array}{l}\text { Deterioro en } \\
\text { autonomía y } \\
\text { ejecución }\end{array}$} & Violencia &, $733^{* *}$ &, $691^{* *}$ &, $735^{* *}$ &, $706^{* *}$ &, $559^{* *}$ &, $675^{* *}$ &, $595^{* *}$ &, $676^{* *}$ \\
\hline & No violencia &, $553^{* *}$ &, $501^{* *}$ &, $515^{* *}$ &, $500^{* *}$ &, $362^{* *}$ &, $417^{* *}$ &, $340^{* *}$ &, $519^{* *}$ \\
\hline Dimensión 3 & Total &, $616^{* *}$ &, $562^{* *}$ &, $559^{* *}$ &, $591^{* *}$ &, $500^{* *}$ &, $509^{* *}$ &, $534^{* *}$ &, $568^{* *}$ \\
\hline \multirow[t]{2}{*}{$\begin{array}{l}\text { Límites } \\
\text { alterados }\end{array}$} & Violencia &, $674^{* *}$ &, $632^{* *}$ &, $644^{* *}$ &, $660^{* * *}$ &, $551^{* *}$ &, $597^{* *}$ &, $601^{* *}$ &, $611^{* *}$ \\
\hline & No violencia &, $489^{* *}$ &, $366^{* *}$ &, $407^{* *}$ &, $418^{* *}$ &, $443^{* *}$ &, $355^{* *}$ &, $389^{* *}$ &, $443^{* *}$ \\
\hline Dimensión 4 & Total &, $562^{* *}$ &, $409^{* *}$ &, $503^{* *}$ &, $507^{* *}$ &, $525^{* *}$ &, $484^{* *}$ &, $499^{* *}$ &, $566^{* *}$ \\
\hline \multirow{2}{*}{$\begin{array}{l}\text { Direccionalidad } \\
\text { hacia los demás }\end{array}$} & Violencia &, $632^{* *}$ &, $545^{* *}$ &, $609^{* *}$ &, $617^{* *}$ &, $543^{* *}$ &, $564^{* *}$ &, $569^{* *}$ &, $628^{* *}$ \\
\hline & No violencia &, $471^{* *}$ &, $233^{* *}$ &, $368^{* *}$ &, $362^{* *}$ &, $495^{* *}$ &, $370^{* *}$ &, $384^{* *}$ &, $483^{* *}$ \\
\hline Dimensión 5 & Total &, $660^{* *}$ &, $553^{* *}$ &, $632^{* *}$ &, $597^{* *}$ &, $529^{* *}$ &, $558^{* *}$ &, $553^{* *}$ &, $633^{* *}$ \\
\hline \multirow{2}{*}{$\begin{array}{l}\text { Sobrevigilancia } \\
\text { e Inhibición }\end{array}$} & Violencia &, $700^{* *}$ &, $618^{* *}$ &, $699^{* *}$ &, $671^{* *}$ &, $563^{* *}$ &, $613^{* *}$ &, $610^{* *}$ &, $677^{* *}$ \\
\hline & No violencia &, $577^{* *}$ &, $413^{* *}$ &, $511^{* *}$ &, $457^{* *}$ &, $493^{* *}$ &, $441^{* *}$ &, $449^{* *}$ &, $531^{* *}$ \\
\hline
\end{tabular}


Cuando se analiza la relación entre los esquemas y la dependencia emocional, en mujeres con violencia, encontramos mayor relación entre la dependencia emocional con los esquemas de desconfianza y abuso $(\mathrm{r}=0.702)$, grandiosidad/ autorización $(\mathrm{r}=0.704)$, fracaso $(\mathrm{r}=0.700)$, dependencia/ incompetencia $(\mathrm{r}=0.680)$, y entre negatividad/ pesimismo ( $\mathrm{r}=0.680$ ); asimismo, los factores de dependencia emocional asociados a los esquemas disfuncionales tempranos son miedo e intolerancia a la soledad $(\mathrm{r}=0.727)$, prioridad de la pareja $(\mathrm{r}=0.713)$ y deseos de control de la pareja $(\mathrm{r}=0.700)$; los cuales hacen evidente que la mujer con violencia en la relación de pareja tiene la expectativa de que será perjudicada, engañada, humillada o que siempre le tocará la peor parte, que debería conseguir lo que ella quiere independientemente de que sea realista o no; sumado a considerarse inepta, incapaz de ser como los demás, propician a que la mujer violentada tiende a guardar o justificar la agresión. Asimismo, desarrollan una sensación de pesimismo y preocupación, temor a que sus vidas se destrocen en cualquier momento si no están alertas permanentemente.

$\mathrm{Al}$ analizar los factores relacionados entre dependencia emocional, el temor a quedarse sola por separación es tan fuerte que en los casos de dependencia emocional grave no se tiene en cuenta si hay menosprecio grave, deterioro manifiesto de la relación e incluso malos tratos. La relación solo se romperá si lo promueve el compañero, y por adversa que haya sido esta relación la dependiente emocional intentará una y otra vez reanudarla, llegando hasta extremos que pueden resultar patéticos para el entorno. En el grupo sin violencia, también existe correlación, aunque menor, y significativa dejando entrever que la presencia de esquemas disfuncionales tempranos asociados a la dependencia emocional si se incrementa una de las variables se corre el riesgo de incrementar la otra.

Finalmente, el encontrar correlación importante, el efecto es significativo en el grupo de mujeres con violencia, lo que indica riesgo latente porque facilita la permanencia en una relación de pareja disfuncional y violenta así como alto riego en su salud mental.

3. Diferencias en las dimensiones de los esquemas cognitivos disfuncionales en las mujeres con y sin violencia en la relación de pareja de la ciudad de Lima. El presente análisis efectuado en mujeres con y sin violencia en la relación de pareja de la ciudad de Lima, se ha contrastado ambos grupos, utilizando el análisis U de Mann Whitney, con el objeto de conocer si difieren en función a las dimensiones en sus esquemas disfuncionales tempranos según la presencia y no de violencia en la relación de pareja encontrando:

a. En la muestra general al analizar los puntajes medios predominan las dimensiones desconexión rechazo (62.77) y sobrevigilancia e inhibición (52.88); cuando hay violencia los puntajes de las dimensiones se incrementan, así las dimensiones que predominan son desconexión rechazo (69.80), sobrevigilancia/inhibición (56.86), deterioro en autonomía y ejecución (54.75) y direccionalidad ante los demás (44.30), hecho que se encuentra en menor nivel cuando no ocurre violencia 
en la relación de pareja como se observa en la dimensión desconexión y rechazo (54.80) y sobrevigilancia e inhibición (48.38), tal como podemos observar en la Tabla 5.

b. En las mujeres con violencia en la relación de pareja, al analizar los puntajes medios de los esquemas que predominan, estos son en mayor número, a saber insuficiente autocontrol/ autodisciplina (15.13), autosacrificio (15.41), normas inalcanzables (14.73), subyugación (14.62), abandono/inestabilidad (14.57), búsqueda de aprobación (14.27), negatividad/pesimismo (14.26), privación emocional (14.25), inhibición emocional (14.22) y desconfianza/abuso (14.18). En cambio, en las mujeres sin violencia son menos los esquemas que se destacan y el indicador es menor, como se observa en el autosacrificio (14.82) e insuficiente autocontrol y disciplina(13.41), tal como se observan en la Tabla 6.

c. Las dimensiones de los esquemas disfuncionales tempranos son mayores cuando hay violencia en la relación de pareja y se torna menor cuando la relación de pareja es sin violencia, como se observa en la Tabla 5.

d. Las dimensiones de los esquemas disfuncionales tempranos más dañados se observan en las mujeres con violencia, desconexión y rechazo, sobre vigilancia e inhibición y deterioro en autonomía y ejecución, lo cual no se destaca en el grupo sin violencia.

e. La dimensión desconexión y rechazo es una constante, cuando hay violencia y se aminora cuando no la hay. Tal como puede verse en la siguiente tabla de estadísticos descriptivos previos a la contrastación. Tabla 5.

Tabla 5. De Diferencia de medias aritméticas de las Dimensiones de los esquemas cognitivos disfuncionales tempranos en mujeres con y sin violencia en la relación de pareja.

\begin{tabular}{llcccc}
\hline Dimensiones & Recuento & Media & $\begin{array}{c}\text { Desviación } \\
\text { estándar }\end{array}$ & Varianza \\
\hline Dimensión 1 & Total & 385 & 62.77 & 22.82 & 520.58 \\
Desconexión y rechazo & Violencia & 204 & 69.80 & 22.42 & 502.55 \\
& No violencia & 181 & 54.86 & 20.61 & 424.85 \\
Dimensión 2 & Total & 385 & 48.97 & 18.79 & 353.13 \\
Deterioro en autonomía y & Violencia & 204 & 54.75 & 18.32 & 335.77 \\
ejecución & No violencia & 181 & 42.45 & 17.15 & 294.16 \\
Dimensión 3 & Total & 385 & 26.54 & 9.64 & 92.93 \\
Límites alterados & Violencia & 204 & 29.25 & 9.26 & 85.70 \\
Dimensión 4 & No violencia & 181 & 23.48 & 9.15 & 83.81 \\
Direccionalidad hacia & Total & 385 & 42.42 & 13.58 & 184.29 \\
los demás & Violencia & 204 & 44.30 & 12.62 & 159.32 \\
Dimensión 5 & No violencia & 181 & 40.29 & 14.31 & 204.88 \\
Sobrevigilancia e & Total & 385 & 52.88 & 18.00 & 324.10 \\
inhibición & Violencia & 204 & 56.86 & 17.60 & 309.73 \\
\hline
\end{tabular}

Tabla 6. Diferencia de medias aritméticas de los 18 esquemas cognitivos disfuncionales tempranos en mujeres con y sin violencia en la relación de pareja. 


\begin{tabular}{lcccccccc}
\hline & \multicolumn{3}{c}{ Violencia } & \multicolumn{5}{c}{ No Violencia } \\
\hline & $\mathrm{N}$ & Media & $\begin{array}{c}\text { Desviación } \\
\text { típ. }\end{array}$ & $\begin{array}{c}\text { Error típ. } \\
\text { de la media }\end{array}$ & $\mathrm{N}$ & Media & $\begin{array}{c}\text { Desviación } \\
\text { típ. }\end{array}$ & $\begin{array}{c}\text { Error típ. } \\
\text { de la media }\end{array}$ \\
\hline Esquema total & 204 & 254.97 & 75.798 & 5.307 & 181 & 209.45 & 70.582 & 5.246 \\
Abandono / inestabilidad & 204 & 14.57 & 5.510 & .386 & 181 & 11.20 & 5.414 & .402 \\
Desconfianza / abuso & 204 & 14.18 & 5.029 & .352 & 181 & 11.41 & 5.412 & .402 \\
Privación emocional & 204 & 14.25 & 4.942 & .346 & 181 & 11.96 & 4.837 & .360 \\
$\begin{array}{l}\text { Imperfección / vergüenza } \\
\text { Aislamiento social / }\end{array}$ & 204 & 13.70 & 4.769 & .334 & 181 & 10.81 & 4.936 & .367 \\
alienación & 204 & 13.11 & 5.167 & .362 & 181 & 9.48 & 4.491 & .334 \\
$\begin{array}{l}\text { Dependencia / } \\
\text { incompetencia }\end{array}$ & 204 & 13.42 & 5.194 & .364 & 181 & 9.72 & 4.931 & .367 \\
$\begin{array}{l}\text { Vulnerabilidad al peligro o } \\
\text { a la enfermedad }\end{array}$ & 204 & 13.38 & 5.008 & .351 & 181 & 10.18 & 4.904 & .364 \\
$\begin{array}{l}\text { Apego confuso y/o } \\
\text { inmaduro }\end{array}$ & 204 & 14.02 & 5.289 & .370 & 181 & 11.34 & 5.542 & .412 \\
Fracaso & 204 & 13.93 & 4.761 & .333 & 181 & 11.22 & 4.885 & .363 \\
$\begin{array}{l}\text { Grandiosidad / } \\
\text { autorización }\end{array}$ & 204 & 13.69 & 5.221 & .366 & 181 & 10.07 & 4.374 & .325 \\
$\begin{array}{l}\text { Insuficiente auto-control/ } \\
\text { auto disciplina }\end{array}$ & 204 & 15.56 & 4.925 & .345 & 181 & 13.41 & 6.009 & .447 \\
$\begin{array}{l}\text { Subyugación } \\
\text { Auto sacrificio }\end{array}$ & 204 & 14.62 & 4.610 & .323 & 181 & 12.70 & 5.546 & .412 \\
$\begin{array}{l}\text { Búsqueda de aprobación } \\
\text { Negatividad / pesimismo }\end{array}$ & 204 & 15.41 & 4.785 & .335 & 181 & 14.82 & 6.019 & .447 \\
$\begin{array}{l}\text { Inhibición emocional } \\
\text { Normas inalcanzables / }\end{array}$ & 204 & 14.27 & 4.604 & .322 & 181 & 12.77 & 5.069 & .377 \\
hipercrítica & 204 & 14.26 & 4.683 & .328 & 181 & 12.17 & 4.710 & .350 \\
Castigo & 204 & 14.73 & 5.116 & .358 & 181 & 12.31 & 5.793 & .431 \\
\hline & & & & .338 & 181 & 12.96 & 5.579 & .415 \\
& 204.66 & 5.034 & .352 & 181 & 10.94 & 4.783 & .355 \\
\hline
\end{tabular}

4. Comparar los factores de la dependencia emocional en las mujeres con y sin violencia en la relación de pareja de la ciudad de Lima.

En el presente análisis efectuado en mujeres con y sin violencia en la relación de pareja de la ciudad de Lima, se han contrastado ambos grupos, utilizando el análisis U de Mann Whitney, con el objeto de conocer si difieren los niveles de dependencia emocional según la presencia o no de violencia en la relación de pareja, y se ha hallado lo siguiente:

a. En la muestra general se observa que los factores más altos son miedo e intolerancia a la soledad (23.06), seguido de miedo a la ruptura (17.87) y prioridad a la pareja (15.98); cuando hay violencia en la relación de pareja, los puntajes se incrementan en los factores miedo e intolerancia a la soledad (25.37), miedo a la ruptura (20.37) y prioridad de la pareja. Hecho que se encuentra en menor nivel cuando no ocurre violencia, apreciándose el factor deseos de exclusividad (20.46) como el que destaca en este grupo, tal como se muestra en la Tabla 7. 
b. En las mujeres con violencia el factor deseos de exclusividad obtiene un promedio de 11.42, a diferencia de las mujeres sin violencia en la relación de pareja en la cual este factor obtiene un promedio de 20.46 .

c. Los factores de la dependencia emocional son mayores cuando hay violencia en la relación de pareja y se vuelve menor cuando la relación de pareja es sin violencia.

Tabla 7. De Diferencia de medias aritméticas de los Factores de dependencia Emocional en mujeres con y sin violencia en la relación de pareja.

\begin{tabular}{|c|c|c|c|c|c|c|c|c|}
\hline & \multicolumn{4}{|c|}{ Violencia } & \multicolumn{4}{|c|}{ No Violencia } \\
\hline & $\mathrm{N}$ & Media & $\begin{array}{l}\text { Desviación } \\
\text { típ. }\end{array}$ & $\begin{array}{l}\text { Error típ. } \\
\text { de la media }\end{array}$ & $\mathrm{N}$ & Media & $\begin{array}{l}\text { Desviación } \\
\text { típ. }\end{array}$ & $\begin{array}{l}\text { Error típ. } \\
\text { de la media }\end{array}$ \\
\hline Dependencia Total & 204 & 113.16 & 38.521 & 2.697 & 181 & 92.25 & 34.957 & 2.598 \\
\hline $\begin{array}{l}\text { Deseos de control y } \\
\text { dominio }\end{array}$ & 204 & 11.85 & 4.485 & .314 & 181 & 15.05 & 6.962 & .517 \\
\hline Deseos de exclusividad & 204 & 11.42 & 4.300 & .301 & 181 & 20.46 & 8.720 & .648 \\
\hline Miedo a la ruptura & 204 & 20.37 & 7.905 & .553 & 181 & 13.91 & 5.997 & .446 \\
\hline $\begin{array}{l}\text { Miedo e intolerancia a la } \\
\text { soledad }\end{array}$ & 204 & 25.36 & 9.258 & .648 & 181 & 13.28 & 5.825 & .433 \\
\hline $\begin{array}{l}\text { Necesidad de acceso a la } \\
\text { pareja }\end{array}$ & 204 & 14.17 & 5.046 & .353 & 181 & 9.64 & 4.558 & .339 \\
\hline Prioridad de la pareja & 204 & 17.82 & 6.759 & .473 & 181 & 10.23 & 4.658 & .346 \\
\hline Subordinación y sumisión & 204 & 12.17 & 4.305 & .301 & 181 & 9.67 & 4.015 & .298 \\
\hline
\end{tabular}

Al contrastar los rangos promedios de los 18 esquemas cognitivos disfuncionales tempranos, utilizando el análisis U de Mann Whitney, se encuentran diferencias significativas entre las mujeres con violencia en la relación de pareja y las sin violencia, en 17 esquemas; no existiendo diferencias significativas, en el esquema autosacrificio en ambos grupos. Las mujeres con violencia presentan mayores rangos medios, como se observa en la Tabla 8 .

En la dependencia emocional, al contrastar los rangos promedios, se encuentra diferencias significativas en los 7 factores entre las mujeres con y sin violencia en la relación de pareja. 
Tabla 8. De contrastación de los Esquemas cognitivos disfuncionales tempranos y la Dependencia emocional en mujeres con y sin violencia en la relación de pareja.

\begin{tabular}{|c|c|c|c|c|c|c|}
\hline & \multicolumn{3}{|c|}{ Violencia } & \multicolumn{3}{|c|}{ No violencia } \\
\hline & $\mathrm{N}$ & $\begin{array}{l}\text { Rango } \\
\text { promedio }\end{array}$ & $\begin{array}{l}\text { Suma de } \\
\text { rangos }\end{array}$ & $\mathrm{N}$ & $\begin{array}{l}\text { Rango } \\
\text { promedio }\end{array}$ & $\begin{array}{c}\text { Suma de } \\
\text { rangos }\end{array}$ \\
\hline Esquemas total & 204 & 224.04 & 45705.00 & 181 & 158.01 & 28600.00 \\
\hline Abandono / inestabilidad & 204 & 226.47 & 46200.50 & 181 & 155.27 & 28104.50 \\
\hline Desconfianza / abuso & 204 & 222.98 & 45487.00 & 181 & 159.22 & 28818.00 \\
\hline Privación emocional & 204 & 217.77 & 44425.50 & 181 & 165.08 & 29879.50 \\
\hline Imperfección / vergüenza & 204 & 226.93 & 46293.50 & 181 & 154.76 & 28011.50 \\
\hline Aislamiento social / alienación & 204 & 231.32 & 47189.50 & 181 & 149.81 & 27115.50 \\
\hline Dependencia / incompetencia & 204 & 232.36 & 47401.00 & 181 & 148.64 & 26904.00 \\
\hline $\begin{array}{l}\text { Vulnerabilidad al peligro o a la } \\
\text { enfermedad }\end{array}$ & 204 & 228.09 & 46530.50 & 181 & 153.45 & 27774.50 \\
\hline Apego confuso y/o inmaduro & 204 & 222.77 & 45444.50 & 181 & 159.45 & 28860.50 \\
\hline Fracaso & 204 & 223.55 & 45604.50 & 181 & 158.57 & 28700.50 \\
\hline Grandiosidad / autorización & 204 & 230.15 & 46951.50 & 181 & 151.12 & 27353.50 \\
\hline $\begin{array}{l}\text { Insuficiente autocontrol / } \\
\text { autodisciplina }\end{array}$ & 204 & 215.74 & 44011.00 & 181 & 167.37 & 30294.00 \\
\hline Subyugación & 204 & 215.52 & 43966.50 & 181 & 167.62 & 30338.50 \\
\hline Autosacrificio & 204 & 199.02 & 40599.50 & 181 & 186.22 & 33705.50 \\
\hline Búsqueda de aprobación & 204 & 210.66 & 42974.50 & 181 & 173.10 & 31330.50 \\
\hline Negatividad / pesimismo & 204 & 217.13 & 44295.50 & 181 & 165.80 & 30009.50 \\
\hline Inhibición emocional & 204 & 206.94 & 42216.50 & 181 & 177.28 & 32088.50 \\
\hline Normas inalcanzables / hipercrítica & 204 & 219.09 & 44694.50 & 181 & 163.59 & 29610.50 \\
\hline Castigo & 204 & 221.59 & 45204.50 & 181 & 160.78 & 29100.50 \\
\hline Dependencia total & 204 & 223.56 & 45606.00 & 181 & 158.56 & 28699.00 \\
\hline Miedo a la ruptura & 204 & 231.67 & 47260.50 & 181 & 149.42 & 27044.50 \\
\hline Miedo e intolerancia a la soledad & 204 & 223.17 & 45526.00 & 181 & 159.00 & 28779.00 \\
\hline Prioridad de la pareja & 204 & 225.75 & 46053.50 & 181 & 156.09 & 28251.50 \\
\hline Necesidad de acceso a la pareja & 204 & 205.66 & 41955.50 & 181 & 178.73 & 32349.50 \\
\hline Deseos de exclusividad & 204 & 217.55 & 44381.00 & 181 & 165.33 & 29924.00 \\
\hline Subordinación y sumisión & 204 & 218.06 & 44485.00 & 181 & 164.75 & 29820.00 \\
\hline Deseos de control y dominio & 204 & 219.62 & 44803.00 & 181 & 162.99 & 29502.00 \\
\hline
\end{tabular}




\section{INTERPRETACIÓN DE LOS DATOS O DISCUSIÓN}

La violencia en la relación de pareja causa distintas consecuencias psicológicas y físicas en sus víctimas, desde síndromes hasta trastornos, siendo las consecuencias más relevantes en el ámbito doméstico el síndrome de adaptación paradójica, depresión, trastornos de ansiedad, trastorno de estrés post traumático (Rincón, 2003; Huerta et al., 2014). Por otro lado, diversas investigaciones (Calvete, 2007; Rincón, 2003) indican que la violencia en el hogar favorece, desde tempranas edades, la instauración de creencias y esquemas mentales disfuncionales en las víctimas de esta, afectando su salud mental y las relaciones interpersonales de todos los miembros de la familia. Young (1999) describe a los esquemas disfuncionales tempranos como una estructura enormemente estable y duradera que se elabora durante la infancia, se desarrolla durante toda la vida del individuo y son disfuncionales en alto grado; esta distorsión cognitiva llevará al sujeto a padecer desadaptación o disfuncionalidad. Así, el estar incluido en una situación de violencia intrafamiliar en la edad adulta favorece la activación de dichos esquemas. En el presente estudio, en la muestra general, encontramos una correlación significativa $(0.01)$ entre las dimensiones de los esquemas con la dependencia emocional. Cuando se analizan los datos en función a la presencia o no de violencia en la relación de pareja, se encuentra en las mujeres con violencia relación muy significativa entre los esquemas cognitivos disfuncionales tempranos y la dependencia emocional; encontrándose mayor relación entre dependencia emocional con los esquemas de desconfianza y abuso, grandiosidad/autorización, fracaso, dependencia/incompetencia, y entre negatividad/ pesimismo; similares resultados obtienen Calvete, Corral y Estévez (2007), en mujeres con historia de maltrato en comparación con las sin maltrato quienes presentaron esquemas cognitivos de apego, desconfianza, grandiosidad, dependencia/incompetencia, estándares inflexibles, fracaso, privación emocional, vulnerabilidad al daño, abandono y autosacrificio. A estos factores se suma el miedo a la soledad y la modificación de planes; datos que indican que existen factores predictivos que aumentan la probabilidad de presentarse violencia en una relación de pareja. Asimismo, en otro estudio realizado por Calvete (2005), observó que las mujeres maltratadas con trastornos como depresión puntúan más alto en esquemas cognitivos relacionados con el autosacrificio, el fracaso, el abandono y la culpa.

Por otro lado, al revisar los factores de dependencia emocional asociados a los esquemas disfuncionales tempranos, en el presente estudio, como miedo e intolerancia a la soledad, prioridad de la pareja y deseos de control de la pareja; estos son similares a los que encuentra Castelló (2005) y Schaeffer (1998), dominio de miedo a la soledad es percibido en la mujeres maltratadas ante el temor a sentir que no son amadas. Las mujeres maltratadas con dependencia emocional necesitan la pareja para sentirse equilibradas y seguras, por lo tanto la soledad es vista como algo aterrador, que siempre le tocará la peor parte, sumado a considerarse incapaz de ser como los demás, propician a que la mujer violentada tiende a guardar o justificar la agresión. La mujer dependiente afectiva emplea más mecanismos de negación y no afrontamiento, distorsionando su evaluación del problema, tal vez 
elaborada como respuesta adaptativa a la situación por la que atraviesa. De este modo, el miedo a la pérdida, a la soledad o al abandono contamina el vínculo afectivo y lo vuelve sumamente vulnerable y patológico, tal como lo investigaron Villa Moral y Sirvent (2009).

Al analizar estos resultados, el efecto es significativo en el grupo de mujeres con violencia en la relación de pareja, lo que indica riesgo latente en la muestra examinada porque facilita la permanencia en una relación de pareja disfuncional y violenta.

Al diferenciar, en las mujeres con violencia, los esquemas que predominan son insuficiente autocontrol/autodisciplina, autosacrificio, normas inalcanzables, subyugación, abandono/ inestabilidad, búsqueda de aprobación, negatividad/ pesimismo, privación emocional (14.25), inhibición emocional (14.22) y desconfianza/abuso (14.18); esquemas que no se encuentran en las mujeres sin violencia. Apreciándose que los esquemas cognitivos disfuncionales tempranos son mayores cuando hay violencia en la relación de pareja y se torna menor cuando la relación de pareja es sin violencia. Situación similar se encuentra al analizar las dimensiones de los esquemas, los más acentuados se observan en las mujeres con violencia, desconexión y rechazo, sobre vigilancia e inhibición y deterioro en autonomía y ejecución, lo cual no se destaca en el grupo sin violencia. Algo similar es reportado por Villegas (2008), señalando que el esquema de abandono en las mujeres maltratadas implica la sensación de que las personas significativas no podrán continuar proporcionando apoyo emocional, vínculos o protección, debido a que son emocionalmente inestables e impredecibles. Dichas creencias expresan el temor y la preocupación de ser abandonados, (Gantiva et al., 2009): las mujeres maltratadas no conciben la idea de ser abandonadas, por lo que se someten a lo que fuera por conservar la presencia de la pareja. Referente al esquema de autosacrificio, Young (1999) plantea que las personas que lo presentan se centran más en satisfacer las necesidades de los demás a expensas de la propias, así las mujeres maltratadas experimentan la sensación de que sus propias necesidades no se satisfacen, y se limitan a satisfacer las necesidades de los demás, aunque todo lo que hagan no sea valorado o tenido en cuenta. Otro estudio realizado por Munera y Tamayo (2013) coincide con los esquemas hallados como autosacrificio, deprivación emocional y abandono, concluyendo que la violencia intrafamiliar favoreció en los sujetos participantes de la investigación la activación de esquemas maladaptativos tempranos con tendencia al abandono y creencias de subyugación, desprotección e insatisfacción de deseos y necesidades.

En lo concerniente a las diferencias en la dependencia emocional entre las mujeres con y sin violencia en la relación de pareja se encuentra que, cuando hay violencia, los puntajes se incrementan en los factores miedo e intolerancia a la soledad, miedo a la ruptura y prioridad a la pareja; resultados que son menores cuando no hay violencia en la relación de pareja. Asimismo, Forcades (2007) señala que la mujer teme la soledad y el hombre la dependencia, la mujer aprecia más el vínculo afectivo que su propia autonomía, y si se ve obligada a elegir, sacrificará su autonomía por 
ese vínculo. En lo referente a la violencia doméstica se ha evidenciado su relación con la dependencia emocional, como encontramos en el presente estudio. Dutton y Painter (1981) consideran que las mujeres maltratadas establecen una relación emocional fuerte denominada unión traumática debido a que uno maltrata al otro y este tiene emociones positivas hacia el que le maltrata; resultados y apreciaciones que también coinciden con lo descrito por Castelló (2005) en mujeres víctimas de malos tratos, encontrando que estas mujeres perciben el miedo a la pérdida, a la soledad, como algo insoportable, asimismo presentan un patrón crónico de demandas afectivas frustradas, que buscan desesperadamente satisfacerse mediante relaciones interpersonales estrechas.

Finalmente, podemos señalar que el estar inserto en una relación de pareja donde hay violencia es un factor de riesgo para la salud mental y la salud en general que favorece la activación de los esquemas y que toda dependencia afectiva supone un comportamiento desadaptativo contingente a una interrelación afectivo dependiente (Sirvent, 2004).

\section{CONCLUSIONES}

- En la muestra total constituida por mujeres con y sin violencia en la relación de pareja existe una relación positiva y significativa entre las 5 dimensiones de los esquemas cognitivos disfuncionales con los 7 factores de la dependencia emocional, predominando las dimensiones desconexión y rechazo; deterioro en autonomía y ejecución y sobrevigilancia e inhibición.

- En el análisis de cada esquema con la dependencia emocional, en la muestra total, existe mayor relación con los esquemas de desconfianza y abuso, privación emocional, fracaso, negatividad/ pesimismo y apego confuso y/o inmaduro; de la misma forma los factores de dependencia emocional asociados son miedo e intolerancia a la soledad, miedo a la ruptura, prioridad de la pareja y deseos de control de la pareja.

- Al relacionar los grupos con y sin violencia, se encuentra una relación positiva y significativa entre las dimensiones de los esquemas cognitivos disfuncionales tempranos y la dependencia emocional, destacando las relaciones entre dependencia emocional con las dimensiones, deterioro de la autonomía y ejecución; con la dimensión desconexión y rechazo; y la dimensión sobrevigilancia e inhibición; seguidas de la relación entre dependencia emocional con las dimensiones límites alterados y direccionalidad ante los demás. En el grupo sin violencia se encuentra también una relación, pero esta es menor y significativa.

- Se encuentra relación entre los esquemas y la dependencia emocional, en las mujeres con violencia, existiendo mayor relación entre dependencia emocional con los esquemas de desconfianza y abuso, grandiosidad/ autorización, fracaso, dependencia/incompetencia, y entre negatividad/ pesimismo; asimismo los factores de dependencia emocional asociados a los esquemas son miedo e 
intolerancia a la soledad, prioridad de la pareja y deseos de control de la pareja; lo que hace evidente que las mujeres con violencia presentan indicadores significativos de dependencia emocional. En el grupo sin violencia, existe correlación menor y significativa.

- En las mujeres con violencia, los esquemas que predominan son, en mayor número, insuficiente autocontrol/autodisciplina, autosacrificio, normas inalcanzables, subyugación, abandono/inestabilidad, búsqueda de aprobación, negatividad/pesimismo, privación emocional, inhibición emocional y desconfianza/abuso; en cambio, en las mujeres sin violencia son menos los esquemas y el indicador es menor.

- Las dimensiones de los esquemas cognitivos disfuncionales tempranos más acentuados se observan en las mujeres con violencia: desconexión y rechazo, sobre vigilancia e inhibición y deterioro en autonomía y ejecución, lo cual no se incide en el grupo sin violencia.

- En mujeres con violencia, los puntajes de dependencia emocional se incrementan en los factores miedo e intolerancia a la soledad, miedo a la ruptura; en las mujeres sin violencia es menor, apreciándose el factor deseos de exclusividad más alto.

- Al contrastar ambos grupos, con y sin violencia, se encuentran diferencias significativas entre las mujeres con y sin violencia en 17 esquemas, no existiendo diferencias en el esquema autosacrificio en ambos grupos. Las mujeres con violencia presentan mayores rangos.

- En la dependencia emocional, se encuentra diferencias significativas, al contrastar los rangos promedios en los 7 factores entre las mujeres con y sin violencia.

\section{REFERENCIAS BIBLIOGRÁFICAS}

Aiquipa, J. (2012). Diseño y Validación del Inventario de dependencia Emocional. Rev. de Investigación en Psicología. 15(1), 133-145.

Agudelo, D., Casadiegos, C., Sánchez, D. (2009). Relación entre esquemas maladaptativos tempranos y características de ansiedad y depresión en estudiantes universitarios. Universitas Psychologica, 8(1), pp. 87-104, Recuperado de http://www.redalyc.org/pdf/647/64712168008.pdf

Calvete, E., Estévez, A. y Corral, S. (2007). Trastorno por estrés postraumático y su relación con esquemas cognitivos disfuncionales en mujeres maltratadas. Psicothema 19 (3), 446-451. Disponible en http://www.psicothema.com/ pdf/3384.pdf

Calvete, E., Corral, S. y Estévez, A. (2007). Violencia contra las mujeres y depresión: Mecanismos cognitivos implicados. Disponible en paginaspersonales. deusto.es/ecalvete/.../violencia-contra-las-mujeres.pdf 
Castrillón, D; et al. (2005). Validación del Yong Schema Questionnaire Long Form - Second Edition (YSQ - L2) en población colombiana. Rev. Latinoam. Psicol..37(.3). Bogotá. Disponible en pepsic.bvsalud.org/scielo. php?script=sci_arttext\&pid=S0120

Dutton, Burghart, Perrin, Chrestman y Halle (1994). Battered women's cognitive schemata. Recuperado en www.ncbi.nlm.nih.gov/pubmed/8012745

Echeverri, J., \& Tamayo, D. (2014). Esquemas maladaptativos tempranos en mujeres víctimas de violencia intrafamiliar, usuarias del Centro de atención a víctimas, Fiscalía, seccional Medellín. Recuperado de revistas.iue.edu.co/ index.php/Psicoespacios/article/download/219/360

García, C. (2014). Dependencia emocional: Una adicción destructiva. Recuperado en psicopedia.org/2588/dependencia-emocional-una-adiccion-destructiva

Huerta, R., et al. (2014). Depresión y ansiedad en mujeres en situación de maltrato en la relación de pareja según tipo de convivencia. Theorema. UNMSM. $1(1)$

Múnera, J., Tamayo, D. (2013). Esquemas maladaptativos tempranos en mujeres víctimas de violencia intrafamiliar, usuarias del C.A.V (Centro de atención a víctimas), Fiscalía, Seccional Medellín. Revista Psicoespacios, 7,(10), pp. 186-202, Disponible en http://revistas.iue.edu.co/index.php/Psicoespacios

Sánchez, G. (2010). Dependencia emocional. Causas, trastorno y tratamiento. Recuperado en www.escuelatranspersonal.com/wp.../dependencia-emocionalgemma.pdf.

Villa Moral, M.; Sirvent, C. (2009). Dependencia afectiva y género: perfil sintomático diferencial en dependientes afectivos españoles. Revista Interamericana de Psicología. Vol. 43, (2) pp. 230-240 España: Fundación Instituto de Drogodependencias "Spiral"

Villegas, M. (2008). Amor y dependencia en las relaciones de pareja, Revista de Psicoterapia. Vol. XVII 68, 5-64. Barcelona, recuperado en ww.herdereditorial.com/media/3450/13.\%20Dependencia.pdf

Walker, L. (2009). El síndrome de la mujer maltratada. Bilbao: Desclée de Brouwer.

Young, J. (1999). Cognitive therapy for personality disorders: A schema-focused approach. Sarasota: Professional Resource Press.

Young, J., Klosko, J., \& Weishaar, M. (2013). Terapia de esquemas. Bilbao: Desclée de Brouwer. 